\title{
Social learning and social transmission of foraging information in Norway rats (Rattus norvegicus)
}

\author{
K. N. LALAND and H. C. PLOTKIN \\ University College London, London, England
}

\begin{abstract}
Adult male Norway rats were tested in a first experiment to see whether foraging efficiency could be improved by social learning. Observers were placed in one of four conditions in which they were paired with demonstrators that either had or had not been previously trained to dig for buried carrot pieces, and in which the demonstrators either did or did not have carrot buried in the experimental enclosure. Observers in the group with trained demonstrators that did have carrot pieces buried in the experimental area during the observation period subsequently unearthed more buried carrot, did so more rapidly, and were generally more active than were the observers in the other three groups. In a second experiment, chains of transmission were established by allowing each observer to act as a demonstrator for the next naive observer. Enhanced levels of digging behavior were maintained across eight transmission episodes in three transmission groups relative to a no-transmission control group, the performance levels becoming stable after five transmission episodes at a level significantly above that of the control group. The study demonstrates that social learning and transmission mechanisms exist which might result in the diffusion of certain patterns of behavior through populations of Norway rats.
\end{abstract}

The experimental study of social learning in rats began with Small's work on puzzle boxes (Small, 1899-1900). It has since been extended to many other situations, encompassing both arbitrary and artificial tasks as well as tasks that bear some relationship to the natural history of the rat (e.g., Bayroff \& Lard, 1944; Galef \& Wigmore, 1983; Miller \& Dollard, 1941; Zajonc, 1965; Zentall \& Levine, 1972). Rats are versatile feeders, eating plants, fungi, various invertebrates such as insects and molluses, and even small vertebrates; yet they are also neophobic (Barnett, 1975; Twigg, 1975). Also, Norway rats in particular inhabit a fixed burrow system, which is used as a base from which to forage (Calhoun, 1962). The social transmission of information about foods, their palatability, location, acquisition, and processing, might partly explain the rat's success as an omnivore. This line of thought has resulted in recent years in the focus of social learning studies of the rat on feeding behaviors (Galef, 1988).

Social learning has already been demonstrated to improve foraging efficiency (Galef \& Wigmore, 1983). What has not been shown is whether behaviors that have been changed by social learning have the potential for becoming fixed in a rat population. For this to occur, social learning must be extended into repeated transmission

We are grateful to C. Heyes of University College London, and to M. Fanselow and the anonymous referees of this journal for helpful comments. This work was carried out under a Science and Engineering postgraduate studentship to $K$. N. Laland. Correspondence should be addressed to H. C. Plotkin at the Department of Psychology, University College London, London WC1E 6BT, England. episodes such that the relevant information can spread through a population.

We had two objectives in the investigation reported here. The first was to establish an experimental situation in which we could be reasonably certain that naive $o b$ server rats can acquire and then act on information gained from the activities of demonstrator conspecifics. The task used was one of digging up buried food. The second objective was to then use such social learning in order to study multiple transmission episodes, by allowing each observer to act as a demonstrator for the next observer. The results of this study may provide some laboratorybased data on the conditions required for the fixation of behaviors in social groups by way of information transmission between animals.

\section{EXPERIMENT 1}

When the behavior of one animal changes as a consequence of its observing the behavior of another animal, there are, broadly, two possible reasons. One, the social learning hypothesis, is that the behavior of the observer animal is altered by way of information about the demonstrator animal's behavior being acquired and stored by the observer. The other, a nonlearning hypothesis, claims that the change in the behavior of the observer occurs without information gain and storage. There are two related means by which this could occur. One is that the motivational state of the observer is transiently altered by the behavior of the demonstrator. The other, known as social facilitation, occurs when the behavior of the demon- 
strator acts as a releaser for the same behavior in the observer (Thorpe, 1956).

This experiment, because the observer animals could act immediately on the basis of the observed behavior of the demonstrator, was designed to distinguish between these explanations. The situation used was one in which observers were placed on one side of an enclosure with food buried beneath the surface. On the other side of the enclosure were demonstrators. The demonstrators were either trained to dig for buried food or not trained to do so. These two conditions were further subdivided on the basis of whether, at the time that the observers and demonstrators were in these neighboring enclosures, food was or was not buried beneath the surface in the demonstrator's section of the enclosure.

The social learning hypothesis predicts that the observers learn that digging leads to the uncovering of buried food, and that digging alone will not significantly alter the behavior of the observers. Thus the animals in the group that observed trained demonstrators digging up buried food should in turn dig up more buried food in their part of the enclosure than should the observers in the other three groups. The nonlearning hypothesis, by contrast, predicts that observers paired with demonstrators previously trained to dig will uncover more buried food than will observers paired with untrained demonstrators, irrespective of whether food was present or absent in the enclosure of the demonstrators at the time that the observers were present. This is because if the behavioral change of the observers were caused by social facilitation, then, since the trained demonstrators will dig more than the untrained demonstrators, digging behavior in the observers will be released irrespective of whether the demonstrator is uncovering food or not. Also, if the digging activities of the demonstrator were somehow altering the motivational state of the observers, causing them to be more active and thus coincidently resulting in more buried food's being uncovered, then, again, the prediction would be that the observers paired with trained demonstrators should dig up more food irrespective of whether or not the demonstrators themselves were digging up food at the time of the pairing.

\section{Method}

Subjects. Sixty-four male rats of an agouti strain were purchased from a commercial dealer, Olac, at 6-7 weeks of age. The experiment was run when the animals were between 10 and 12 weeks of age and weighed between 196 and $222 \mathrm{~g}$. They were housed in pairs, each pair subsequently to be a demonstrator-observer pair, in standard laboratory cages enriched with a variety of play objects. They received ad-lib food and water and were on a 12:12-h light:dark cycle.

Apparatus. The animals were trained and tested in plastic walled enclosures measuring $52 \times 36 \times 18 \mathrm{~cm}$ with stainless steel wire roofs. The enclosures were divided along their length by an $18-\mathrm{cm}-\mathrm{high}$ stainless steel wire mesh of $6 \mathrm{~mm}^{2}$ gauge. The floor covering varied according to group and phase of testing, and was either a 5-cm layer of lightly compressed peat, or a $0.2-\mathrm{cm}$ scattering of peat. The buried food comprised $1 \times 1 \times 0.5 \mathrm{~cm}$ pieces of carrot distributed randomly but either buried beneath the $5-\mathrm{cm}$ depth of peat or placed upon the surface. A pilot study had shown that carrot buried beneath $5 \mathrm{~cm}$ of peat could not be detected without digging.

Procedure. The animals were randomly assigned to one of four groups, with 8 rats serving as demonstrators in each group and 8 as observers. For 14 consecutive days, the demonstrators were placed in the plastic enclosures for $10 \mathrm{~min}$. In two of the groups, the demonstrators had a $5-\mathrm{cm}$ covering of peat on the floor, beneath which were buried 16 carrot pieces. These were the trained demonstrators. On the average, these animals dug up 6 pieces of carrot per day across this training period. The demonstrators in the other two groups were placed in enclosures with the light scattering of peat on the floor and with 6 pieces of carrot placed at random upon the surface. These were the untrained demonstrators. Thus all demonstrators consumed roughly the same amount of carrot during this phase of the experiment, but half of them did not learn to dig in order to gain access to the carrot. The observers were also placed in test enclosures with a light scattering of peat on the floor for the same 10 -min period, but without any carrots in the enclosures. They were fed 6 pieces of carrot on return to their home cages.

On the 15 th day, the animals were placed for $10 \mathrm{~min}$ in the test apparatus as observer-demonstrator pairs, each animal on one side of the wire mesh barrier where observation of the demonstrators by the observers could occur. The floor covering for all observers was $5 \mathrm{~cm}$ of peat, beneath which were buried 16 pieces of carrot. The trained demonstrators were subdivided such that half of them had the $5-\mathrm{cm}$ peat floor covering with 16 buried pieces of carrot (the trained-carrots-available group) and half had the same peat surface without buried carrot pieces (the trained-without-carrotsavailable group). The untrained demonstrators were also subdivided such that half of them were placed in enclosures with carrot buried beneath $5 \mathrm{~cm}$ of peat (the untrained-carrots-available group) and half were placed in enclosures with a $5-\mathrm{cm}$ peat floor covering but no buried carrot (the untrained-without-carrots-available group).

The number of carrots dug up, the time for each to be dug up, and the time of onset of digging behavior in the observer animals was recorded, as it was for demonstrator animals that had carrots available. Also recorded were the general activity levels of all demonstrator and observer animals on a scale of $1-5$, covering a range from complete inactivity (1), through some ambulation but no digging, to vigorous digging and rapid ambulation (5), each animal rated four times in the session. The experiment was conducted blind, the experimenter not knowing which groups the animals belonged to at test.

\section{Results and Discussion}

The number of carrots dug up by each group of observers is shown in Figure 1. An analysis of variance found significant differences on this measure $[F(3,28)=4.95$, $p<.05]$, with observers from the trained-carrotsavailable group digging up approximately twice as many carrots as did observers in any of the other groups. It should be noted that the demonstrators in the trainedcarrots-available group dug up significantly more carrots during the observation phase of the experiment $(X=7.87)$ than did the demonstrators in the untrained-carrotsavailable group $(\bar{X}=2.88)[t(14)=8.87, p<.001]$.

The observers in the trained-carrots-available group also unearthed the first carrot more quickly $(\bar{X}=204 \mathrm{sec})$ than did observers in the other groups $(\bar{X}=305 \mathrm{sec})$. Because group variances differed by a factor greater than four, these data were tested for significance using a nonparametric test and found to be significant [Wilcoxon rank sum test; $W_{s}=92, W(8,24)<93, p<.05$, one-tailed]. 


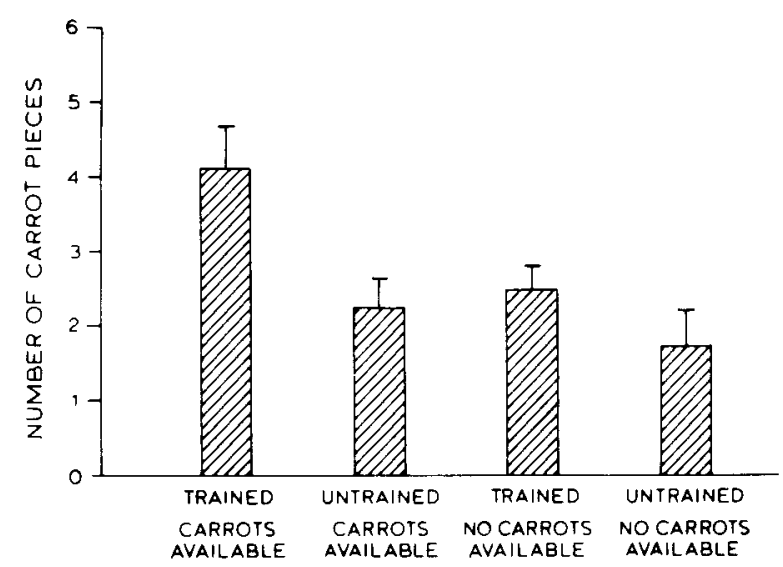

Figure 1. Experiment 1: Mean number of carrot pieces dug up by observers in each group.

The demonstrators in the trained-carrots-available group $(\bar{X}=108 \mathrm{sec})$ dug up their first carrot significantly faster $[t(14)=4.12, p<.01]$ than did the demonstrators in the untrained-carrots-available group $(\bar{X}=255 \mathrm{sec})$.

The observers in the trained-carrots-available group also began digging earlier $(\bar{X}=51 \mathrm{sec})$ than the observers in the other three groups $(\bar{X}=130 \mathrm{sec})$ [Wilcoxon rank sum test; $W_{s}=84.5, W(8,24)<86, p<.025$, one-tailed]. An analysis of variance of time to onset of digging in the demonstrators was also significant $[F(3,28)=$ $6.58, p<.05]$; the mean for the trained demonstrators was $33 \mathrm{sec}$ and that for the untrained demonstrators $129 \mathrm{sec}$.

Figure 2 shows the activity measured for both observers and demonstrators in all four groups. An analysis of variance on the data for the observers showed significant differences $[F(3,28)=4.26, p<.05]$, the observers with trained demonstrators exhibiting higher activity levels $(\bar{X}=14.4)$ than observers with untrained demonstrators $(\bar{X}=10.8)[F(1,28)=8.7, p<.01]$. The demonstra-

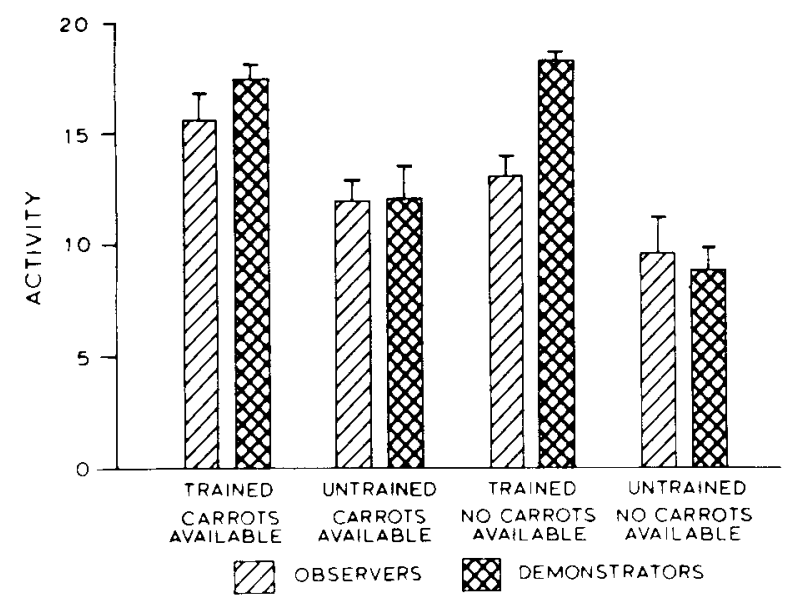

Figure 2. Experiment 1: Mean level of activity for observers and demonstrators in each group. tors themselves showed significantly different levels of activity, those that were trained scoring higher than those that were untrained $[F(1,28)=62.07, p<.01]$. It is of interest to note that there was also a significant difference between the activity of untrained demonstrators, the presence of carrots raising the level of activity $[F(1,28)=$ $5.93, p<.05]$.

Anecdotally, observers whose demonstrators had carrots present appeared to be more goal-directed in their digging behavior, tending to dig deeper in specific areas, with the digging action involving the movement of front legs towards the body. Observers whose trained demonstrators had no carrots in the enclosure showed a greater tendency to dig "wildly," flitting from place to place, shifting mainly surface peat, and often digging with movements of the front legs away from the body. The observers in the untrained-without-carrots-available group often exhibited long periods of inactivity.

The pattern of results is consistent across measures and clearly supports the social learning hypothesis. The observers from the trained-carrots-available group consistently performed better than the observers from the other groups, despite the fact that the activity levels of the demonstrators from the trained-without-carrots-available group were equal to those of the demonstrators of the trained-carrots-available group. The latter finding should have led to similar performance by observers in these two groups, were either form of the nonsocial learning hypothesis correct, but it did not.

On the basis of these findings, we tentatively conclude that in the situation reported here, foraging efficiency is enhanced when social learning operates between pairs of animals.

\section{EXPERIMENT 2}

A demonstration of social learning is not sufficient grounds for the conclusion that social transmission of sufficient penetration to result in the diffusion of a behavior throughout a population will inevitably occur. If, for example, Animal $C$ learns from Animal B less well than Animal B learned from Animal A, and if Animal D learns from Animal $C$ worse still, then transmission might break down rapidly. Instances of behavioral diffusion among animals have been reported in several field studies; examples include the spread of food processing techniques among Japanese macaques (Kawai, 1965) and black rats (Ewer, 1971), the opening of milk bottles by birds (Fisher \& Hinde, 1949), and the feeding behavior of chickadees (Krebs, 1973) and pigeons (Lefebvre, 1986). Field studies, however, do not readily lend themselves to a proper analysis of the mechanism of the spread of a behavior, and it is especially difficult to be certain that the transmission has occurred by way of social learning (Galef, 1980; Sherry \& Galef, 1984). Lefebvre and colleagues have circumvented these problems by combining laboratory, field, and aviary studies to investigate the transmission of food-finding behavior in feral pigeons 
(Giraldeau \& Lefebvre, 1986, 1987; Lefebvre, 1986; Palameta \& Lefebvre, 1985), while Curio has carried out laboratory studies to investigate the social transmission of enemy recognition in birds (Curio, Ernst, \& Vieth, 1978; Vieth, Curio, \& Ernst, 1980).

In Experiment 2, we extended this approach to the rat. Experiment 1 led us to conclude that the carrot-digging task could be acquired by social learning. We could then broaden the design of the previous experiment to one in which each observer would act as a demonstrator for another animal, to test whether or not social learning could result in social transmission.

\section{Method}

Subjects. The subjects were $\mathbf{2 8 0}$ rats identical in all respects to those described for Experiment 1.

Apparatus. The apparatus was the same as that described for Experiment 1.

Procedure. The animals were randomly assigned to one of four groups. Three of these were social transmission groups in which each animal first had the role of the observer of a demonstrator conspecific that had varying degrees of experience at foraging by digging. The observer then became a demonstrator for the next animal in the line of transmission. In this way, it might be possible for information to be transmitted from the initial demonstrator, through intermediate observer-demonstrator animals, to the last observer in the chain. In the first group, the standard transmission group, the first demonstrator was trained to dig for carrots beneath the peat surface of the enclosures. Thereafter, each observer then served as a demonstrator for another animal. In the second group, the additional individual learning group, each observer was given a further period in which to dig for carrots on its own before acting as a demonstrator for the next animal. In the third group, the innovator group, the initial demonstrator animal was untrained. The fourth group was the control, in that no social transmission could occur, and so there was no transmission chain. Each observer was paired with an untrained demonstrator that had no carrots buried on its side of the enclosure, and thus each animal performed on the basis of the occurrence of its own individual learning in the presence of a naive conspecific.

For the first 14 days of the experiment, every subject was placed in an enclosure for $10 \mathrm{~min}$. For the animals that were subsequently to serve as trained demonstrators for the standard transmission and additional individual learning groups, the surface was covered by $5 \mathrm{~cm}$ of lightly compressed peat, beneath which were buried 16 pieces of carrot. All other animals were placed in enclosures with a light scattering of peat and without carrots. The latter animals all received 6 pieces of carrot on being returned to their home cages.

Testing took place on Days 15-18, each transmission chain being completed in $150 \mathrm{~min}$. For the standard transmission group, the first observer $\left(\mathrm{O}_{1}\right)$ was removed from its home cage and placed in the enclosure with a previously trained demonstrator, each on one side of the dividing partition. The floor surface for each animal was covered with $5 \mathrm{~cm}$ of lightly compressed peat, beneath which were buried 16 pieces of carrot. After $10 \mathrm{~min}$, the demonstrator was removed and the observer placed in a holding cage for $10 \mathrm{~min}$, where there was free access to water but no food. Animal $\mathrm{O}_{1}$ was then returned to a fresh enclosure, now with the next animal, $\mathrm{O}_{2}$, with buried carrots present on each side of the enclosure, and their behavior was recorded for $10 \mathrm{~min} . \mathrm{O}_{1}$ was then removed, and $\mathrm{O}_{2}$ placed in the holding cage for $10 \mathrm{~min}$. This was continued for Animals $\mathrm{O}_{2}$ and $\mathrm{O}_{3}$, and on to $\mathrm{O}$, and $\mathrm{O}_{8}$. The entire transmission chain procedure, with its eight transmission episodes, was repeated eight times to give an $n=8$.

The procedure for the animals in the additional individual learning group was the same as that for the standard transmission group. but for one difference. Each observer was not placed in a holding box for the 10-min period between successive transmission episodes, but was allowed to continue digging in the same test enclosure after the demonstrator had been removed, before being placed with the next observer animal in a fresh enclosure. Thus, these animals had the opportunity to add to their foraging experience through their own individual learning before acting as demonstrators. The procedure for subjects in the innovator group was identical to that for the standard transmission group, except that the demonstrator at the start of each transmission chain was untrained in digging for carrots. In the control group, there was no transmission chain, and though the performances of successive animals were independent of each other, the individual testing episodes were separated by the same 10 -min interval

\section{Results and Discussion}

A two-way (groups $\times$ stage in transmission chain) analysis of variance on the number of carrot pieces dug up gave a significant main effect for groups $[F(3,192)=$ $37.12, p<.011$. There were no differences between the standard transmission and additional individual learning groups. But both unearthed significantly more carrot pieces than did the innovator group $[F(1,192)=58.7$, $p<.01]$, and all three of the transmission groups dug up more carrot pieces than did the control group $[F(1,192)=$ $82.69, p<.01]$. There was also a significant interaction between the groups and the stage of transmission $[F(21,192)=2.41, p<.01]$. Linear trend analysis showed significant downward trends in carrot-digging performance with increasing stage of transmission for the standard transmission group $[F(1,192)=15.17, p<.01]$ and the group with additional individual learning $[F(1,192)=7.00, p<.05]$, as well as a significant upward trend for the innovator group $[F(1,192)=20.18$, $p<.01]$. These data are presented in Figure 3.

This general picture for the observers was supported by an analysis of variance conducted on the numbers of carrot pieces dug up by the animals as demonstrators, where the main groups effect was significant $[F(2,147)=21.80, p<.01]$. Demonstrators in the standard transmission group dug up more carrots $(X=3.3)$ than did demonstrators in both the additional individual

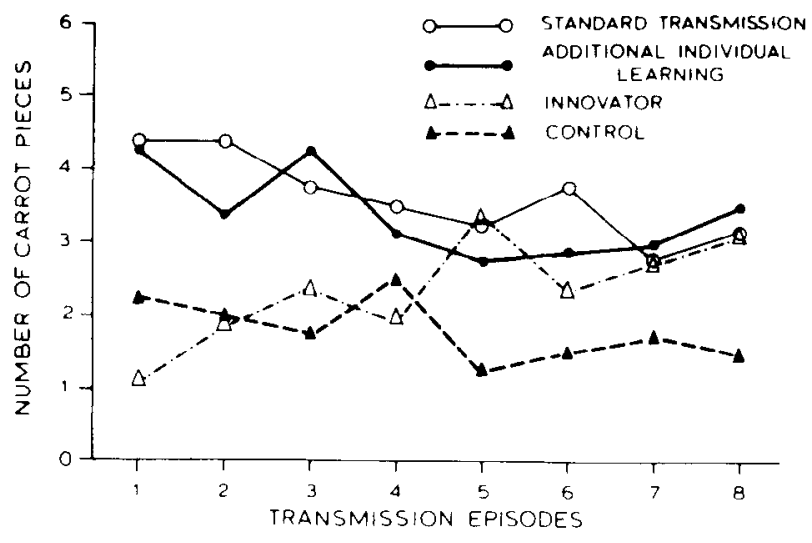

Figure 3. Experiment 2: Mean number of carrot pieces dug up by observers in each group for each step in the transmission chain. 
learning group $(\bar{X}=2.3)$ and the innovator group $(\bar{X}=1.9)$. There was also a significant interaction between groups and stage of transmission $[F(12,147)=2.51$, $p<.05]$. A linear trend analysis showed a significant downward trend for carrot digging performance of demonstrators in the standard transmission group $[F(1,147)=$ $17.83, p<.01]$ and a significant upward trend for the innovator group $[F(1,147)=14.53, p<.01]$. There was no significant trend for the additional individual learning group.

There was a significant linear regression of demonstrator on observer performance for all three transmission groups $[b=0.34, r=0.48, F(1,190)=58.27, p<$ $.01]$. There were also significant correlations between the performance of individual animals as observers and as demonstrators for the standard transmission group $[r=0.59, t(54)=5.37, p<.01]$ and the innovator group $[r=0.41, t(54)=3.30, p<.01]$, but not for the additional individual learning group $(r=0.06)$. The performance of animals as observers and then as demonstrators is shown in Figure 4. The animals in the standard transmission and innovator groups were on the average slightly worse as demonstrators than as observers ( $\bar{X}_{\text {observer }}-\bar{X}_{\text {demonstrator }}=0.31$ and 0.45 , respectively), whereas animals in the group with additional individual learning showed a much larger drop in their performance as demonstrators when compared to their performance as observers $\left(\bar{X}_{\text {observer }}-\bar{X}_{\text {demonstrator }}=1.09\right)$.

Observers in all the transmission groups commenced digging earlier $(\bar{X}=29,36$, and $39 \mathrm{sec}$ for the standard transmission, additional individual learning, and innovator groups, respectively) than did the animals in the control group $(\bar{X}=95 \mathrm{sec})[F(3,192)=15.22, p<.01]$. A linear regression of the demonstrators' onset of digging time on that of the observers' found that $53 \%$ of the variability in the observers' times is directly predictable from the variability in the demonstrators' times $[r=0.73$, $\left.r^{2}=0.53, F=254.98, p<.001\right]$.

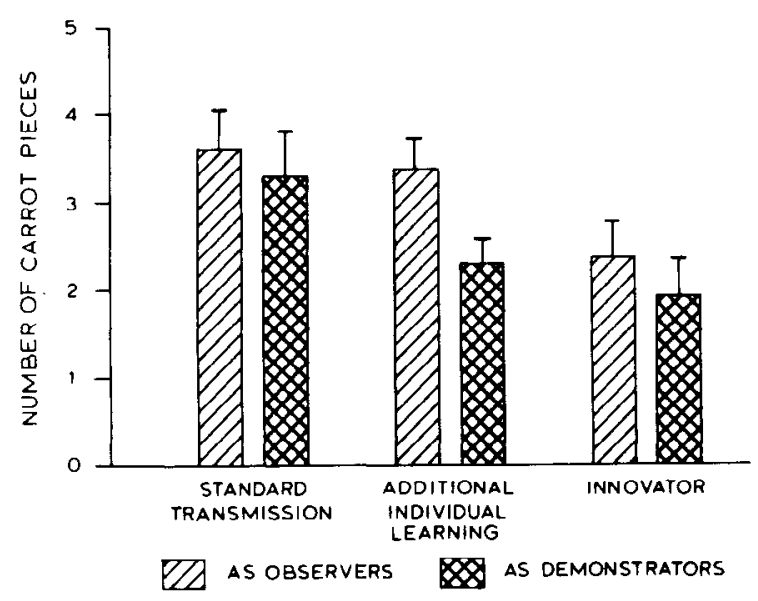

Figure 4. Experiment 2: Mean number of carrot pieces dug up by rats in the three transmission groups both as observers and then as demonstrators.
Although there was no difference between the activity of observers in the standard transmission and in the additional individual learning groups $(\bar{X}=16.4$ and 15.5 , respectively), there were significant differences between these two groups and the innovator group $(\bar{X}=14.3)$ $[F(1,192)=11.29, p<.01]$, and also between the three transmission groups and the control group $(\bar{X}=10.8)$ $[F(3,192)=23.35, p<.01]$. Linear trend analysis showed an upward trend for the activity of both observers $[F(1,192)=8.67, p<.05]$ and demonstrators $[F(1,147)=10.73, p<.01]$ in the innovator group. It is of interest to note that when the animals were demonstrators, those in the standard transmission group $(\bar{X}=15.4)$ were more active than the demonstrators in the additional individual learning group $(\bar{X}=12.4)$ and the innovator group $(\bar{X}=12.5)$. A linear regression of demonstrators' activity on that of the observers showed that $35 \%$ of the variability in the observers' scores was directly predictable from the variability in the activity of the demonstrators $\left[r=0.59, r^{2}=0.35, F=88.27\right.$, $p<.01]$. Also, the activity of animals in both the standard transmission and innovator groups was slightly less when they were demonstrators than when they were observers $\left(\bar{X}_{\text {observers }}-\bar{X}_{\text {demonstrators }}=0.98\right.$ and 1.77 , respectively), whereas animals in the additional individual learning group showed a much larger drop in their activity as demonstrators, when compared with their activity as observers $\left(\bar{X}_{\text {observers }}-\bar{X}_{\text {demonstrators }}=3.12\right)$.

The results of Experiment 2 are also relatively consistent and show that social transmission of a behavior pattern can occur among Norway rats. The enhanced levels of digging for buried food were maintained across eight transmission episodes for all three transmission groups, a finding that is supported by the significant linear regressions that allow the prediction of observer performance from demonstrator performance, with strong correlations between them.

The animals in the standard transmission and additional individual learning groups were more active, began digging earlier, and dug up more pieces of carrot more quickly than did the animals in either the innovator or the control group. There was a decreasing trend in carrotdigging efficiency across the early stages of the transmission chain, but this seemed to stabilize by the fifth transmission episode at a level significantly above that of the control group. This pattern is consistent with the interpretation of an initial loss of information at each transmission episode, resulting in each successive animal's being a less effective demonstrator for the next animal in the line of transmission; this loss then seemed to be counterbalanced beyond a certain point by some other factor that maintained the behavior at levels above those expected when transmission was absent.

The innovator group, so called because the transmission chain began with an untrained demonstrator and because whatever information was being transmitted between animals was the result of some form of cumulative innovation, also reached performance levels of foraging 
efficiency that were significantly better than those for the control group. In the case of this group, though, the trend was toward an improvement in behavior across the transmission episodes from the early stages, when their behavior was similar to that of the controls, to the later stages when it was significantly better than the behavior of the controls and indistinguishable from the behavior of the other transmission groups. Thus, for this group, there seemed to be an accumulation of information within the chain. It is of some interest that the behavior of this group converges on that of the other two transmission groups. The results from the innovator group suggest that the loss of information that occurs with each transmission episode can indeed be offset by the enhancement of the behavior of the observer by the sum of the demonstrator's social and individual learning.

The reason for running the group with additional individual learning was the expectation that such additional learning as might occur would buttress the social learning and lead to the maintenance of higher performance levels across the chain of transmission episodes. Not only did this not occur, but there is some indication in the data, especially in the drop in activity levels between the animals as observers and subsequently as demonstrators, that the additional experience detracted from their effectiveness as demonstrators. The reason for this is not clear, but it may be connected with the timing of the individual learning period. Further experiments are needed, in which the intervals between the interpolated individual learning experience and the observer-demonstrator stages are manipulated. It should also be noted that motivational factors such as fatigue, thirst, or satiation cannot account for either the differences found between the groups, or the trends shown across the transmission chains. These factors would have tended to reduce the differences between the groups and to counteract the trends within groups.

Taken together, both experiments fit well with the growing literature suggesting that social learning and social transmission play an important role in the foraging and feeding behavior of Norway rats (Galef, 1988). The study also addresses the issue of the relative roles of individual and social learning in the spread of behaviors between conspecifics (Boyd \& Richerson, 1985; Plotkin, 1988). The eight transmission episodes described in Experiment 2 are a very modest number, and there is no evidence yet as to whether or not social interactions between freely interacting rats might block transmission, as has been reported for pigeons (Giraldeau \& Lefebvre, 1987). Thus it would be premature to claim that these studies demonstrate that social transmission can result in the fixation within a population of novel behavioral patterns. This experiment does, however, suggest the kinds of experiments that need to be done in the future to provide adequate empirical support for theories of behavioral diffusion.

\section{REFERENCES}

BARNeTt, S. A. (1975). The rat: A study in behavior. Chicago: University of Chicago Press.

BAYROFF, A. G., LARD, K. E. (1944). Experimental social behavior of animals: III. Imitation leaming of white rats. Joumal of Comparaive \& Physiological Psychology, 51, 327-333.

Boyd, R., Richerson, P. J. (1985). Culture and the evolutionary process. Chicago: University of Chicago Press.

CALHoun, J. B. (1962). The ecology and sociology of the Norway rat. (Public Health Publication No. 1008). Bethesda, MD: U.S. Department of Health

Curio, E., Ernst, U., \& VieTh, W. (1978). The adaptive significance of avian mobbing: II. Cultural transmission of enemy recognition in blackbirds. Zeitschrift für Tierpsychologie, 48, 184-202.

EWER, R. F. (1971). The biology and behaviour of a free-living population of black rats (Rattus rattus). Animal Behaviour Monographs, 4, 127-174.

FisHeR, J., HINDE, R. A. (1949). The opening of milk bottles by birds. British Birds, 42, 347-357

GALEF, B. G. (1980). Diving for food: Analysis of a possible case of social learning in wild rats (Rattus norvegicus). Journal of Comparative \& Physiological Psychology, 94, 416-425

GALEF, B. G. (1988). Communication of information concerning distant diets in a social, central place foraging species, Rattus norvegicus. In T. R. Zentall \& B. G. Galef (Eds.), Social learning (pp. 119139). Hillsdale, Erlbaum.

Galef, B. G., Wigmore, S. W. (1983). Transfer of information concerning distant food sites: A laboratory investigation of the "information-centre" hypothesis. Animal Behaviour, 31, 748-758.

Giraldeau, L. A., \& Lefebvre, L. (1986). Exchangeable producer and scrounger roles in a captive flock of feral pigeons. Animal Behaviour, 34, 797-803.

Giraldeau, L. A., \& Lefebvre, L. (1987). Scrounging prevents cultural transmission of food-finding behaviour in pigeons. Animal Behaviour, 35, 387-394.

KAWAI, M. (1965). Newly acquired pre-cultural behaviour of the natural troop of Japanese monkeys on Koshima Inlet. Primates, 2, 1-30.

KREBS, J. R. (1973). Social leaming and the significance of mixed-species flocks of chickadees (Parus spp). Canadian Journal of Zoology, 51, 1275-1288.

Lefebvre, L. (1986). Cultural diffusion of a novel food-finding behaviour in urban pigeons: An experimental field test. Ethology, 71, 295-304.

Miller, N. E., \& Dollard, J. (1941). Social learning and imitation. New Haven, CT: Yale University Press.

Palameta, B., \& Lefebvre, L. (1985). The social transmission of a food-finding technique in pigeons: What is learned? Animal Behaviour, 33, 892-896.

Plotkin, H. C. (1988). Learning and evolution. In H. C. Plotkin (Ed.), The role of behavior in evolution (pp. 133-164). Cambridge, MA: MIT Press.

Sherry, D. F., \& GaleF, B. G. (1984). Cultural transmission without imitation: Milk bottle opening by birds. Animal Behaviour, 32 , 937-938

SMALL, W. S. (1899-1900). An experimental study of the mental processes of the rat. American Journal of Psychology, 11, 133-165.

THORPE, W. H. (1956). Learning and instincts in animals. London: Methuen.

TwIGG, G. (1975). The brown rat. London: David \& Charles.

VIETH, W., CURIo, E., ERNST, U. (1980). The adaptive significance of avian mobbing: III: Cultural transmission of enemy recognition in blackbirds; cross-species tutoring and properties of learning. Animal Behaviour, 28, 1217-1229.

Zajonc, R. B. (1965). Social facilitation. Science, 149, 269-274.

Zentall, T. R., Levine, J. M. (1972). Observational learning and social facilitation in the rat. Science, 170, 1220-1221. 